\section{Resistenzsituation in Europa und Deutschland - Wie groß sind die Probleme?}

\author{
T. Eckmanns ${ }^{1}$, I. Noll ${ }^{1}$ \\ 1 Robert Koch-Institut, Berlin
}

Die Probleme sind groß. Auf höchster Ebene werden Strategien zur Begrenzung der weiteren Ausbreitung von Antibiotikaresistenzen gefordert und entwickelt. Das Weltwirtschaftsforum hat 2013 und 2014 die Antibiotikaresistenz als eine der großen möglichen Gefahren für die weltweite wirtschaftliche Entwicklung eingeschätzt. Im Mai 2014 wurde im renommierten Journal Nature die Gründung eines Weltrates zum Thema Antibiotikaresistenz entsprechend des Weltklimarates angeregt. Im September 2014 hat die US-Regierung eine nationale Strategie zur Bekämpfung von antibiotikaresistenten Bakterien herausgebracht, wesentliche Punkte sind Intensivierung von Stewardship, Surveillance und Forschung. Die Weltgesundheitsorganisation (WHO) hat dieses Jahr erstmals einen Bericht herausgegeben, der auf der Basis von Daten aus 114 Ländern eine globale Bestandsaufnahme gibt. Es wird deutlich, dass die Resistenzsituation in vielen Entwicklungsländern alarmierend ist. Das Resistenzniveau dort ist wesentlich höher als in den meisten Industrienationen. Kaum Diagnostik und dadurch ungerichteter Einsatz von Antibiotika sind entscheidende Ursachen dafür. Für 2015 ist von der WHO ein globaler Aktionsplan angekündigt, der allen relevanten Akteuren klare Aufgaben zuordnen soll.

Für die Mitgliedsstaaten der Europäischen Union existiert das Surveillancesystem European Antimicrobial Resistance Surveillance Network (EARS-Net), koordiniert vom European Center for Disease Prevention and Control (ECDC), das seit 1998 Daten zur Resistenz der klinisch bedeutsamsten Erreger aus Blutkulturen erhebt und damit die Beschreibung zeitlicher Trends und regionaler Unterschiede ermöglicht.

$\mathrm{Zu}$ Beginn der Surveillance standen die gram-positiven Erreger im Fokus, insbesondere die Methicillin-Resistenz von Staphylococcus aureus (MRSA) sowie die Resistenz gegenüber Vancomycin von Enterococcus faecium (VRE). In vielen Ländern Europas hat sich die Situation hinsichtlich MRSA stabilisiert bzw. sogar positiv entwickelt. So sank der Anteil der MRSA an allen S. aureus in den letzten vier Jahren in sieben Ländern, darunter Deutschland, signifikant; 2013 betrug er in Deutschland 15\%. Der Anteil der VRE an allen E. faecium ist in EU-Europa recht stabil, in Deutschland stieg er jedoch signifikant an und lag 2012 bei $16 \%$ und damit im EU-europäischen Vergleich an vierter Stelle.

Bei den gram-negativen Erregern ist in den letzten vier Jahren auf breiter Basis eine Zunahme der Resistenz gegenüber verschiedenen Substanzklassen in Europa zu verzeichnen. So ist bei Escherichia coli und Klebsiella (K.) pneumonia ein kon- tinuierlicher Anstieg der Mehrfach-Resistenz gegenüber 3. Generations-Cephalosporinen, Fluorchinolonen und Aminoglykosiden in mehr als einem Drittel der berichtenden Länder in den letzten Jahren zu sehen. Die Resistenz gegenüber Carbapenemen ist in der Breite noch sehr gering, markante Ausnahmen sind Griechenland mit einem Anteil von Carbapenem-resistenten K. pneumoniae 2013 von 60,5\%, Italien $(28,8 \%)$ und Zypern (9,2\%). Ein alarmierendes Beispiel ist die Entwicklung in Italien, die einen sprunghaften Anstieg in einem relativ kurzen Zeitraum zeigt: von 1,3\% im Jahr 2009, über 15,2\% im Folgejahr auf 26,7\% 2011. In Deutschland liegt der Anteil Carbapenem-resistenter K. pneumoniae noch unter $1 \%$, aber es gibt immer wieder zum Teil sehr große Ausbrüche mit Carbapenem-resistenten K. pneumoniae. Bei Pseudomonas aeruginosa erreicht die Mehrfach-Resistenz gegenüber mindestens 3 von 5 Substanzen bzw. Substanzklassen (Piperacillin, Ceftazidim, Fluorchinolone, Carbapeneme und Aminoglykoside) EU-europaweit ein Niveau von 15\%, Deutschland liegt bei $8,4 \%$.

In Deutschland existieren verschiedene Surveillancesysteme zur Erfassung von Erregern und deren Resistenz: 2008 wurde ARS - Antibiotika-Resistenz-Surveillance in Deutschland - als nationales Surveillancesystem am Robert Koch-Institut (RKI) etabliert, das sowohl die stationäre Krankenversorgung als auch den Sektor der ambulanten Versorgung abdeckt. ARS ist als laborgestütztes Surveillancesystem zur kontinuierlichen Erhebung von Resistenzdaten aus der Routine für das gesamte Spektrum klinisch relevanter bakterieller Erreger konzipiert. 2013 erstreckte sich die Datenbasis von ARS auf Daten von über 400 Krankenhäusern und über 7000 Praxen. Referenzdaten sind über eine interaktive Datenbank öffentlich zugänglich (https://www.ars.rki.de); für die häufigsten bakteriellen Erreger können Berichte zur Resistenzsituation und -entwicklung in der stationären bzw. ambulanten Versorgung generiert werden, die regional und nach Strukturmerkmalen (Krankenhaustyp, Fachrichtung) differenziert werden können.

Das Krankenhaus-Infektions-Surveillancesystem (KISS) des Nationalen Referenzzentrums für Surveillance von nosokomialen Infektionen folgt einem anderen Ansatz: Hier werden krankenhaus- bzw. stationsbezogen die Auftretenshäufigkeiten von Erregern von nosokomialen Infektionen sowie von multiresistenten Erregern erfasst.

Die positive Entwicklung bei MRSA hat gezeigt, dass die Einführung und Umsetzung von Maßnahmen und das gemeinsame Handeln verschiedener Akteure erfolgreich sein kann. Die Entwicklung und Ausbreitung von Resistenzen gegenüber antimikrobiellen Substanzen ist ein multifaktorielles Geschehen. Unter anderem spielen Antibiotikaverbrauch, Hygienemanagement, Diagnostik und Surveillance in der Humanmedizin, aber auch Antibiotikaeinsatz in Veterinärmedizin und Tierhaltung, der Eintrag von Antibiotika in die Umwelt sowie das Reiseverhalten eine Rolle. Nur interdisziplinäres Handeln kann zum Erfolg führen.

Interessenkonflikte: Es bestehen keine Interessenkonflikte. 


\section{Literatur}

1 WHO. Antimicrobial resistance. Global Report on Surveillance 2014

2 World Economic Forum. Global risks 2014, 9. Edition

3 Woolhouse M, Farrar J. An intergovernmental panel on antimicrobial resistance. Nature 2014; 509: S55

4 The White House. National Strategy for Combating Antibiotic-resistant Bacteria, September 2014

5 ECDC. Surveillance report. Antimicrobial resistance surveillance in Europe; 2012

6 Im Internet: https://www.ars.rki.de; Stand: September 2014

\section{Bibliografie}

Dol http://dx.doi.org/10.1055/s-0033-1358026

Drug Res 2014; 64, Suppl. 1: S3-S4

(c) Georg Thieme Verlag KG Stuttgart · New York . ISSN 2194-9379

\section{Korrespondenzadresse}

Tim Eckmanns

Robert Koch-Institut

Seestraße 10

13353 Berlin

eckmannst@rki.de 